\title{
Optimal Design of Low Pass Filter by Rectangular, Taylor and Hamming Technique
}

\author{
Tarun Kumar Chandra ${ }^{1}$, Gautam Sharma ${ }^{2}$, Pranay Kumar Rahi ${ }^{3}$ \\ B.E. Scholar, Dept of Electrical \& Electronics Engineering, Institute of Technology Korba, Chhattisgarh, India ${ }^{1,2}$ \\ Asst Professor, Dept of Electrical \& Electronics Engineering, Institute of Technology Korba, Chhattisgarh, India ${ }^{3}$
}

\begin{abstract}
Digital filters are widely used in many Digital Signal Processing applications. Therefore, Digital Filtering is one of the basic needs of Digital Signal Processing. This paper introduces the definition and basic principles of FIR digital filters, and the design based on MATLAB. After the description of the process of design., the MATLAB program is Used to implement FIR filter using modified coefficient of Hamming window function and also calculate the equivalent noise bandwidth. Digital filters are pervasive in the present era of communication systems. As a result good digital filter performance is important and hence to design a digital finite impulse response (FIR) filter satisfying all the required condition is a demanding one. This report deals with the design of FIR digital filter using hamming window technique. This window is optimized to minimize the maximum (nearest) side lobe, giving it a height of about one-fifth that of the Hamming window. Hence this type of filter plays very important role in spectral analysis of different types of signal. In spectral analysis applications, a small main lobe width of the window function in frequency domain is required for increasing the ability to distinguish two closely spaced frequency components.
\end{abstract}

Keywords: FIR Filter, DSP, FFT, Rectangular Window, Taylor Window, Hamming Window, MATLAB.

\section{INTRODUCTION}

The digital signal processing has become an extremely important subject. A fundamental aspect of Digital Signal Processing is filtering. A digital filter is a system that performs mathematical operations on a sampled, discrete-time signal to reduce or enhance certain aspects of that signal. Digital filters with finite duration impulse response referred to as FIR filters [1]. FIR filters are non-recursive type filters where by the present output depends on present input and past inputs. FIR filters are widely used than IIR filters, because FIR digital filters have an exactly linear phase, always stable, non-recursive structure and arbitrary amplitude-

FIR filter is described by the difference equation.

$\mathrm{y}(\mathrm{n})=\sum_{k=0}^{n-1}(h(k) x(n-k))$

Where $\mathrm{x}(\mathrm{n})$ is the input signal and $\mathrm{h}(\mathrm{n})$ is the impulse response of FIR system [2].

The most popular and widely used window functions are; Rectangular window, Hanning window, Hamming window and Kaiser Window. The Rectangular window response provides side lobes which gives rise to ripples in pass band and stop band. Advanced digital signal processing systems require specialized high-performance embedded computer architectures. The term high-performance translates to large amounts of data and computations per time unit. The term embedded further implies requirements on physical size and power efficiency. Thus the requirements are of both functional and non-functional nature. Digital filter is essentially a system or network that improves quality of a signal and/or extracts information from the signals or separates two or more signals which are previously combined. Digital filters are used in numerous applications e.g. control system, system for audio and video processing and communication systems. Now a day's digital filters can be used to perform many filtering tasks are replacing the traditional role of Analog filters in many Applications. Digital filters can be applied to very low frequency signals, such as those occurring in biomedical and seismic applications very efficiently. In addition, the characteristics of digital filters can be changed or a adapted by simply changing the content of a finite number of registers, thus multiple filters are usually used to discriminate a frequency or a band of frequencies from a given signals which is normally a mixture of both desired and undesired signals[3]. These are mainly two types of filter algorithms. They are finite impulse response filter (FIR) and infinite impulse response filter (IIR). In case of a FIR filter, the response due to an impulse input will decay with in a finite time. But for IIR filter, the impulse response never dies out. FIR filters are commonly known as non-recursive filters and IIR filters are known as recursive filters. The impulse response is „finite" because there is no feedback in the filter if put in an impulse (that is, a single "1" sample followed by many "0" samples), zeroes will eventually come out after the "1" sample has made its way in the delay line past the entire coefficient. 
FIR filter design using Hamming window function provides smaller main lobe width and sharp transition band. This type of filter is very useful in spectral analysis of different types of signals [4].

\section{WINDOWING TECNIQUE}

The Windowing method involves multiplying the ideal impulse response with a window function to generate a corresponding filter, which tapers the ideal impulse response. Like the frequency sampling method, the windowing method produces a filter whose frequency response approximates a desired frequency response. The windowing method, however, tends to produce better results than the frequency sampling method.

In the time domain windowing means to multiply the infinite impulse response $\mathrm{hD}(\mathrm{n})$ by a finite duration window function $\mathrm{w}(\mathrm{n})$ to get a truncation. The resulted impulse response $\mathrm{h}(\mathrm{n})$ is their product and is given as follows:

$\mathrm{H}(\mathrm{n})=\mathrm{hD}(\mathrm{n}) * \mathrm{w}(\mathrm{n}), 0 \leq \mathrm{n} \leq \mathrm{M}$

The basic idea behind the Window method of filter design is that the ideal frequency response of the desired filter is equal to 1 for all the pass band frequencies, and equal to 0 for all the stop band frequencies and then the filter impulse response is obtained by taking the Discrete Fourier Transform (DFT) of the ideal frequency response. Unfortunately, the filter response would be infinitely long since it has to reproduce the infinitely steep discontinuities in the ideal frequency response at the band edges. To create a Finite Impulse Response (FIR) filter, the time domain filter coefficients must be restricted in number by multiplying by a window function of a finite width $[5,6]$.

\subsection{HAMMING WINDOW}

Syntax: $\mathrm{w}=$ hamming $(\mathrm{L})$

Description: It returns an L-point symmetric Hamming window in the column vector w. L should be a positive integer. The coefficients of a Hamming window are computed from the following equation. Hamming window is optimized to minimize the maximum side lobe. It is defined by

$$
w[n]=\left\{\begin{array}{cc}
0.54-0.46 \cos \left(\frac{2 \pi n}{M}\right), & \begin{array}{c}
0 \leq n \leq M \\
\text { other }
\end{array} \\
0 &
\end{array}\right.
$$

\subsection{RECTANGULAR WINDOW}

Syntax: $\mathrm{w}=$ rectwin $(\mathrm{L})$

Description: It returns a rectangular window of length $\mathrm{L}$ in the column vector w. This function is provided for completeness; a rectangular window is equivalent to no window at all. It is defined by:

$$
w[n]=\left\{\begin{array}{l}
1,0 \leq n \leq M \\
0, \text { other }
\end{array}\right.
$$

\subsection{TAYLOR WINDOW}

Syntax: $\mathrm{w}=$ taylorwin $(\mathrm{n})$

Description: A Taylor window allows to make tradeoffs between the mainlobe width and sidelobe level. The Taylor distribution avoids edge discontinuities. Taylor windows are typically used in radar applications, such as weighting synthetic aperature radar images and antenna design. It returns an n-point Taylor window in a column vector w. The values in this vector are the window weights or coefficients. $n$ must be a positive integer [7].

\section{COMPARISON OF FIR AND IIR}

(1) Under the same conditions as in the technical indicators, output of the IIR filter has feedback to input, so it can meet the requirements better than FIR. The storage units are less than those of IIR, the number of calculations is also less, and it's more economical.

(2) The phase of FIR filter is strictly linear, while the IIR filter is not. The better the selectivity of IIR filter is, the more serious the nonlinearity of the phase will be [8].

(3) The FIR filter is non-recursive structure, finite precision arithmetic error is very small. While IIR filter is recursive structure, and parasitic oscillation may occur in the operation of IIR filter. 
(4) Fast Fourier Transformation can be used in FIR filter, while IIR cannot.

(5) The IIR filter can make use of the formulas, data and tables of the analog filter, and only a small amount of calculation. While FIR filter design may always make use of the computer to calculate, and the order of FIR filter could be large to meet the design specifications [9].

\section{DESIGN SIMULATION}

To design the low pass FIR filter using MATLAB Hamming, Rectangular and Taylor Window. The parameter Specification are given in table 1 . As

Table (1). Parameter specification

\begin{tabular}{|c|c|}
\hline PARAMETER & VALUE \\
\hline Sampling Frequency(fx) & $48000 \mathrm{~Hz}$ \\
\hline Cutt Off Frequency(fc) & $10800 \mathrm{~Hz}$ \\
\hline Order $(\mathrm{N})$ & 10 \\
\hline
\end{tabular}
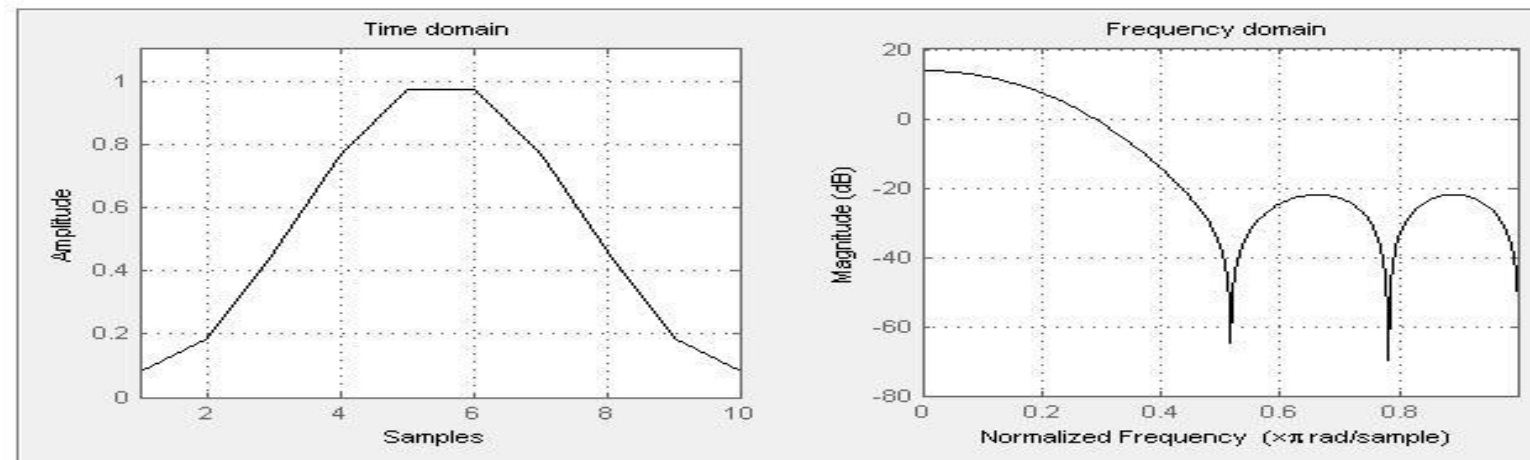

Figure (1): Time domain and Frequency domain of Hamming window

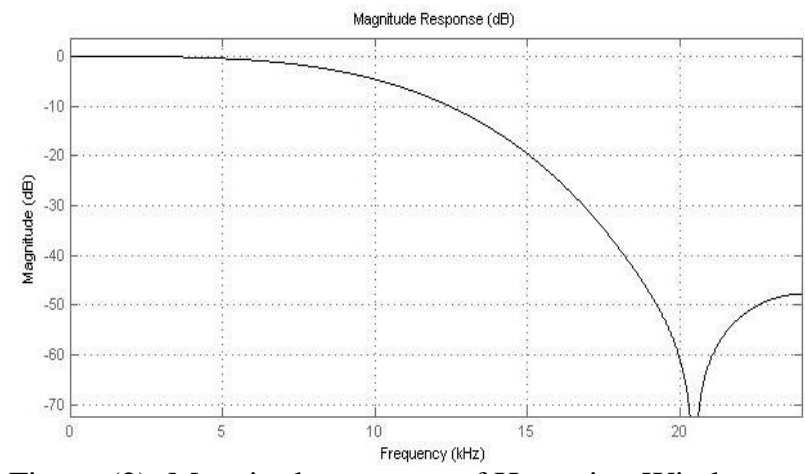

Figure (2): Magnitude response of Hamming Window

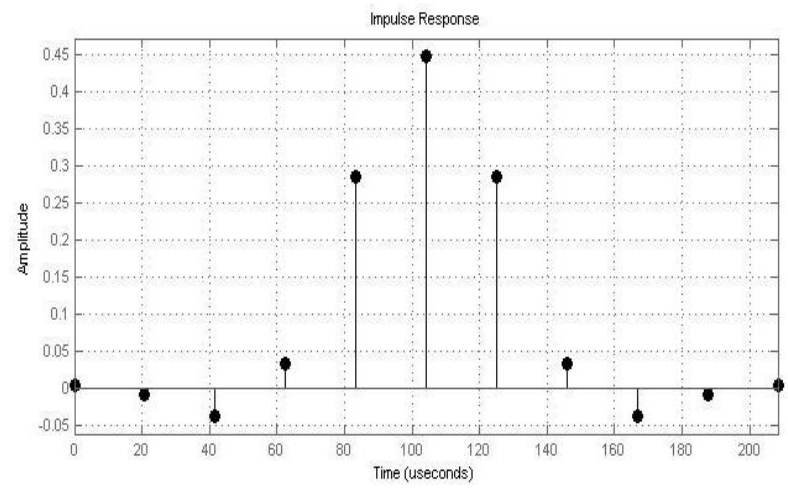

Figure (4): Impulse response of Hamming Window

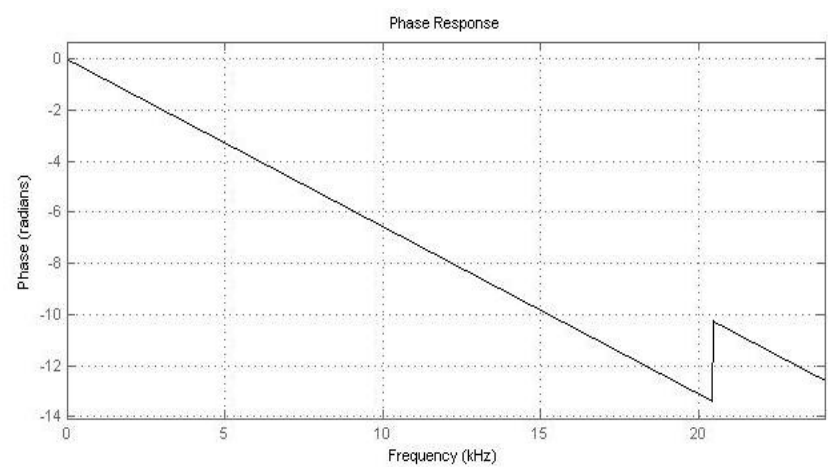

Figure (3): Phase response of Hamming Window

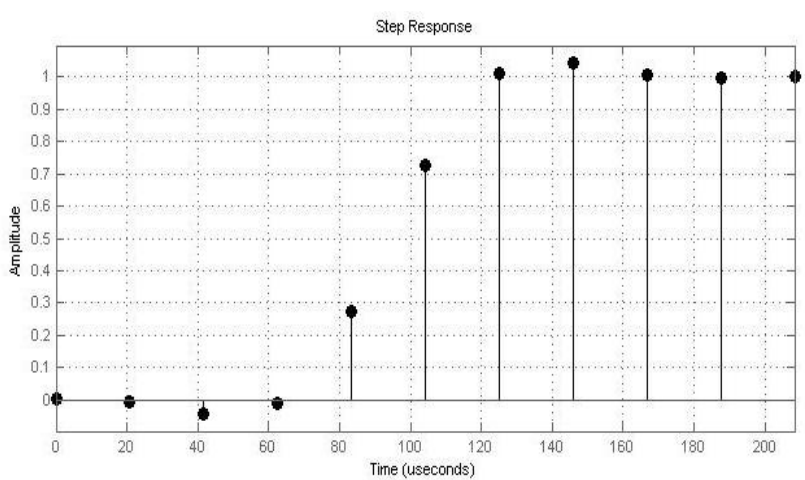

Figure (5): Step response of Hamming Window 


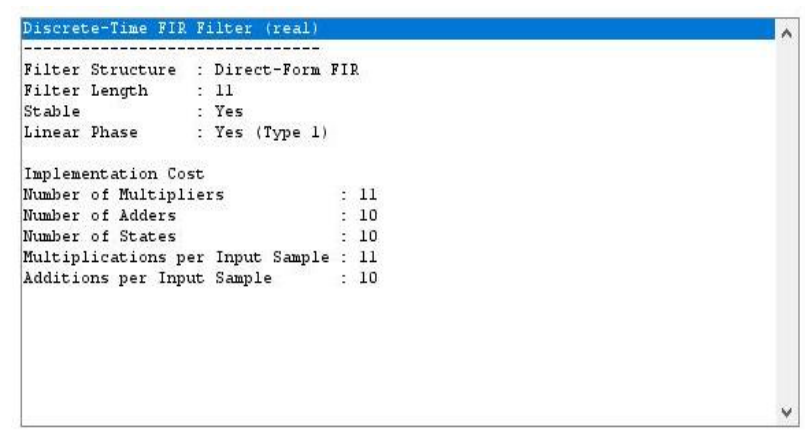

Figure (6): Filter coefficient of Hamming Window

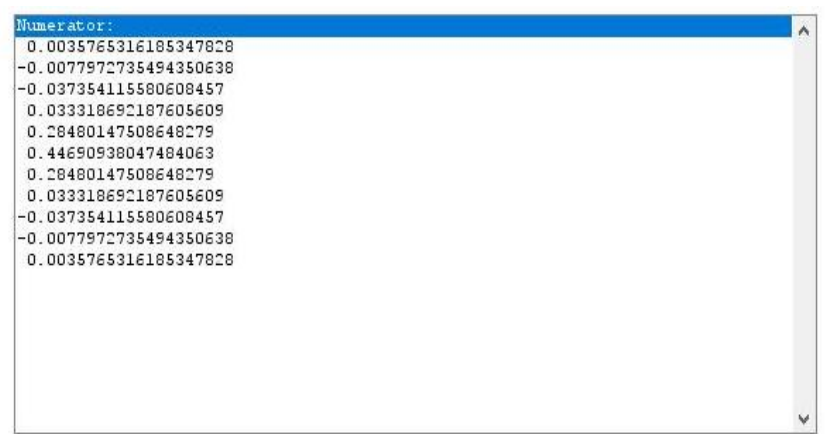

Figure (7): Information of Hamming Window

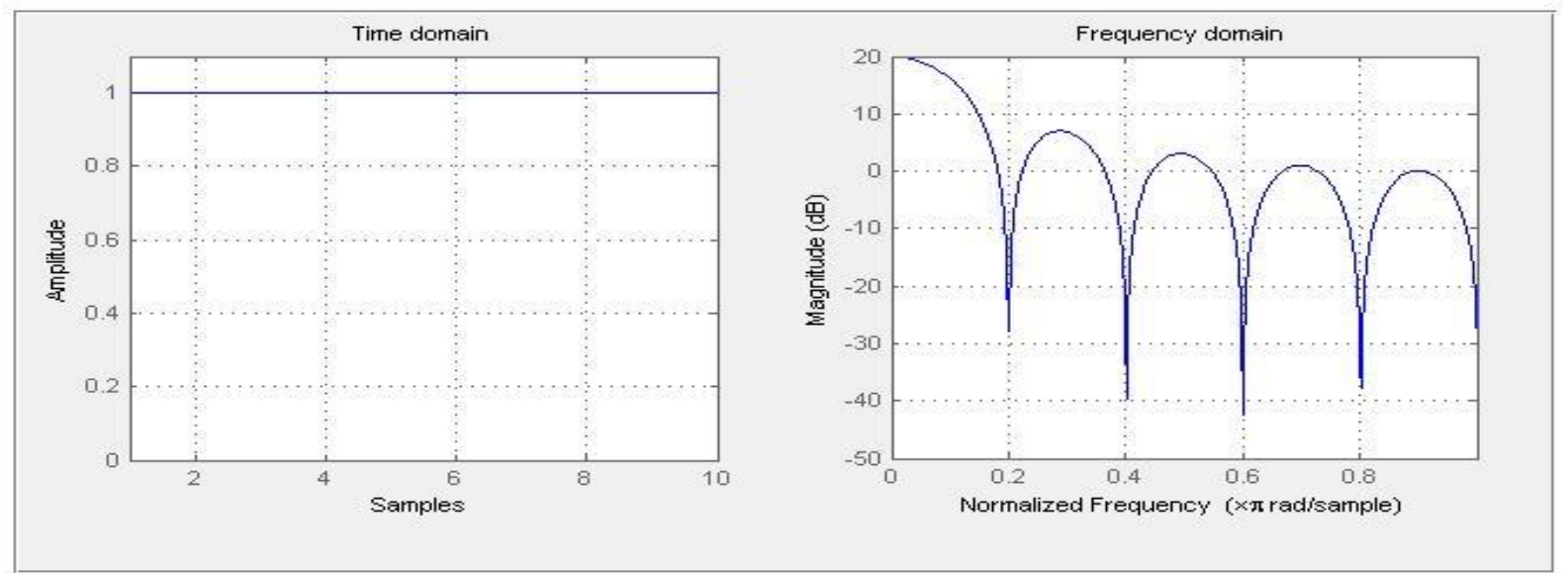

Figure (8): Time domain and Frequency domain of Rectangular window

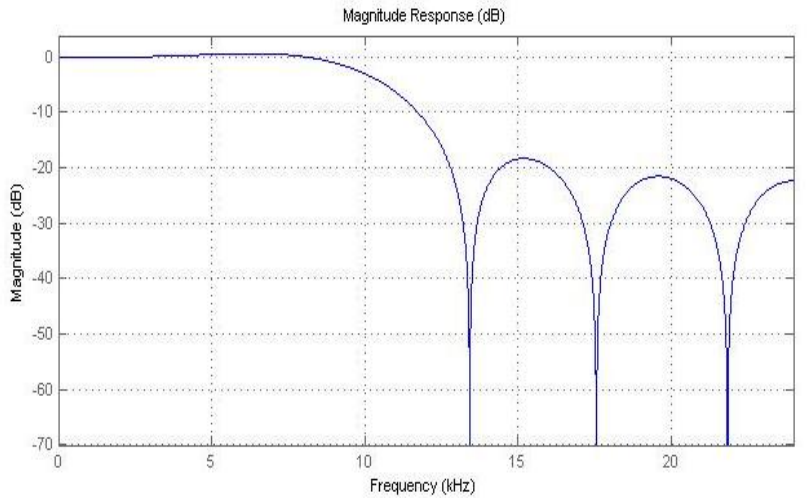

Figure (9): Magnitude response of Rectangular Window

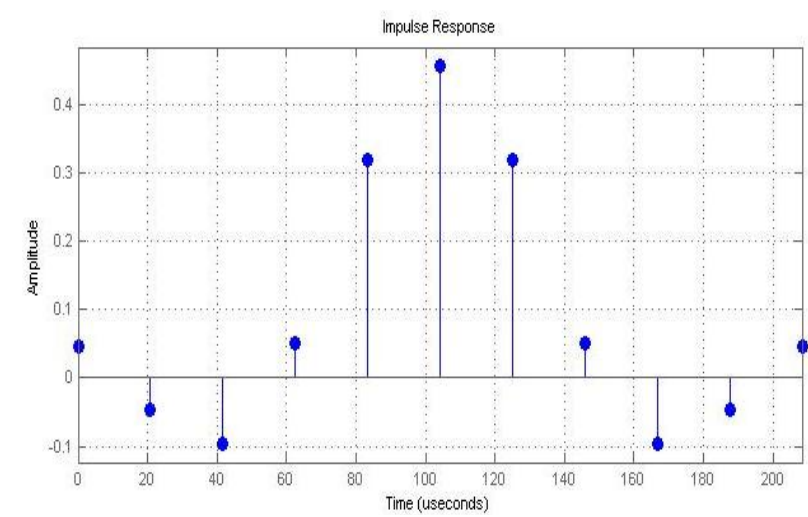

Figure (11): Impulse response of Rectangular Window

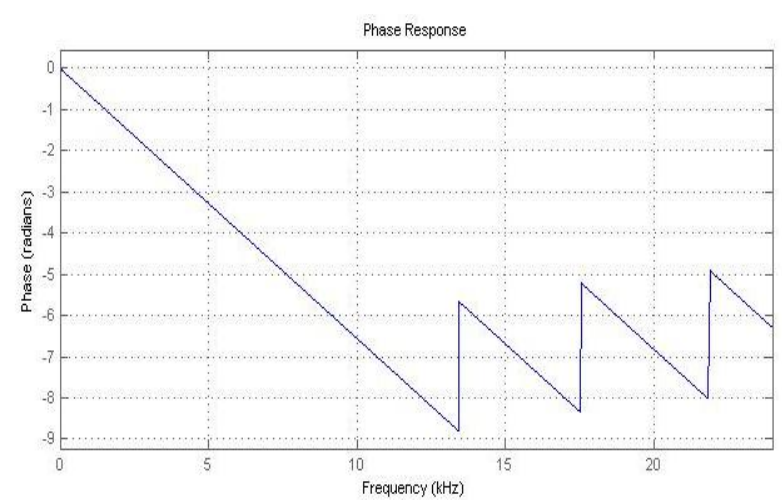

Figure (10): Phase response of Rectangular Window

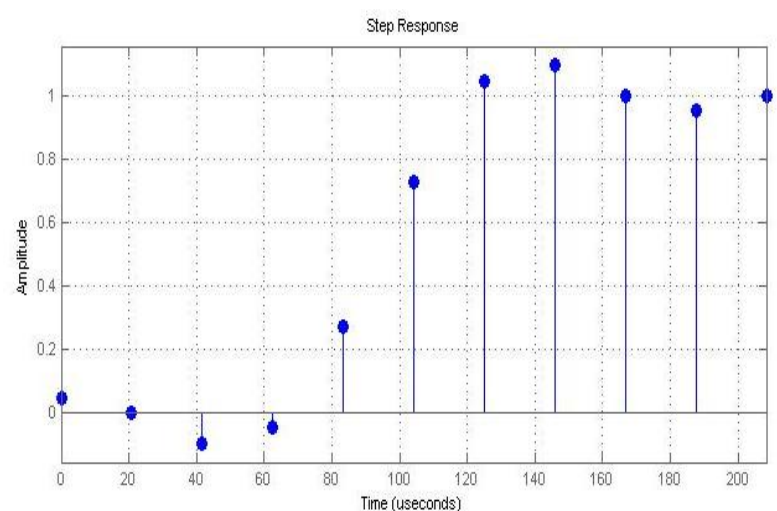

Figure (12): Step response of Rectangular Window 


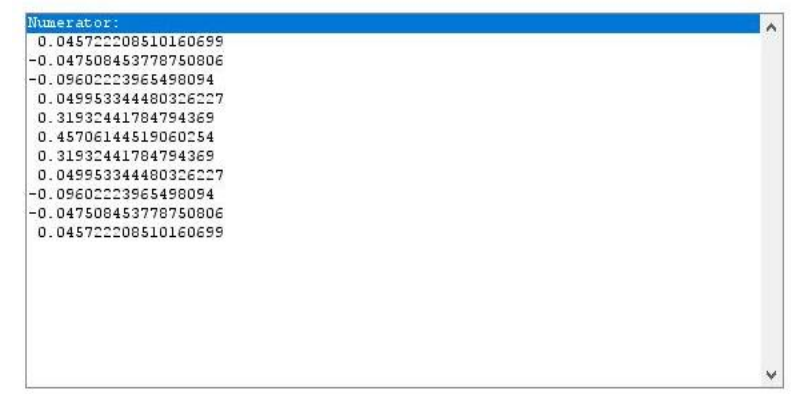

Figure (13): Filter coefficient of Rectangular Window

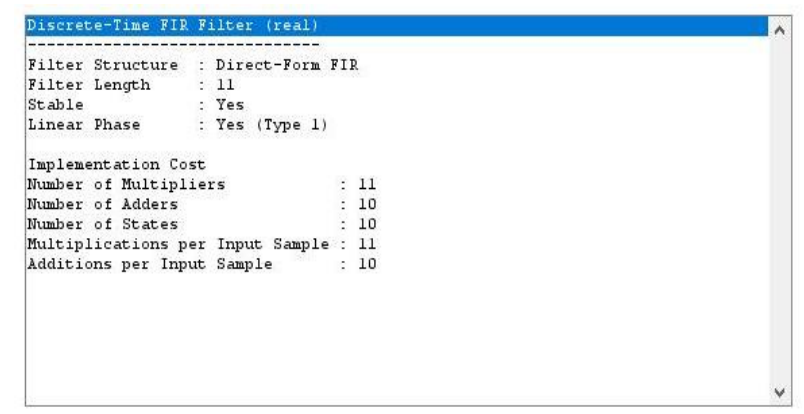

Figure (14): Information of Rectangular Window
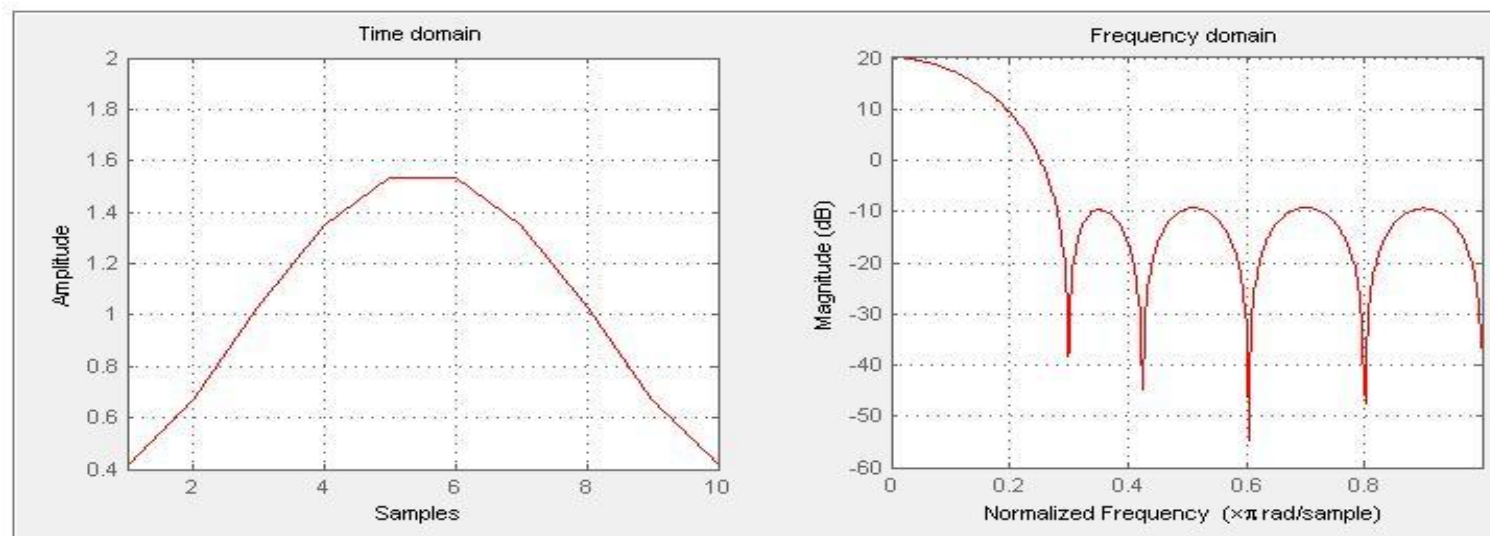

Figure (15): Time domain and Frequency domain of Taylor window

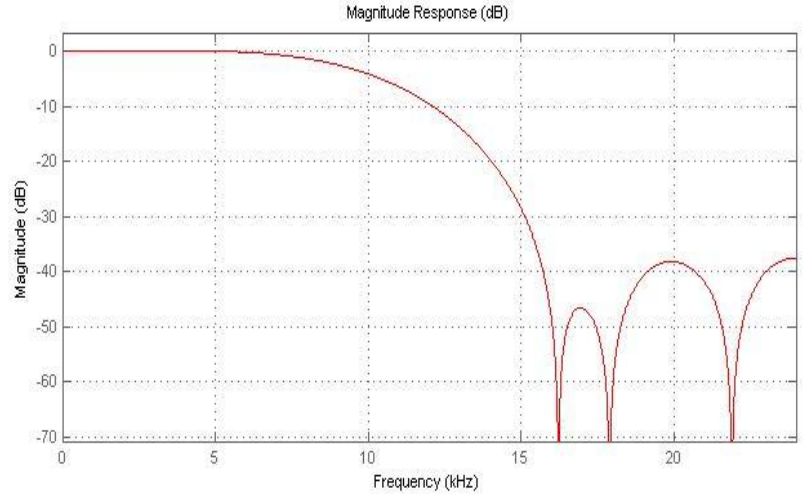

Figure (16): Magnitude response of Taylor Window

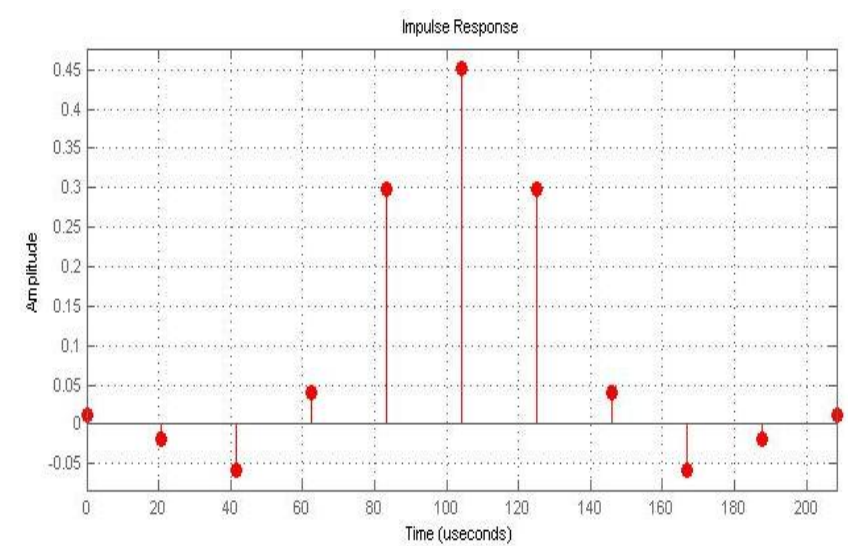

Figure (18): Impulse response of Taylor Window

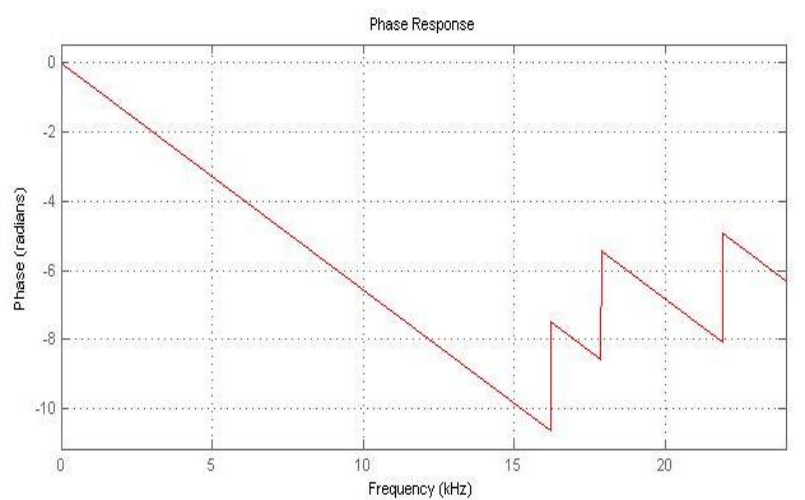

Figure (17): Phase response of Taylor Window

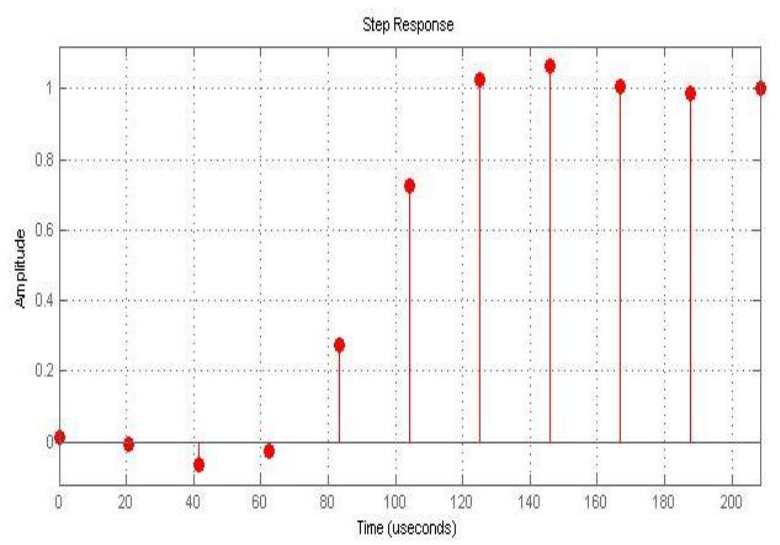

Figure (19): Step response of Taylor Window 


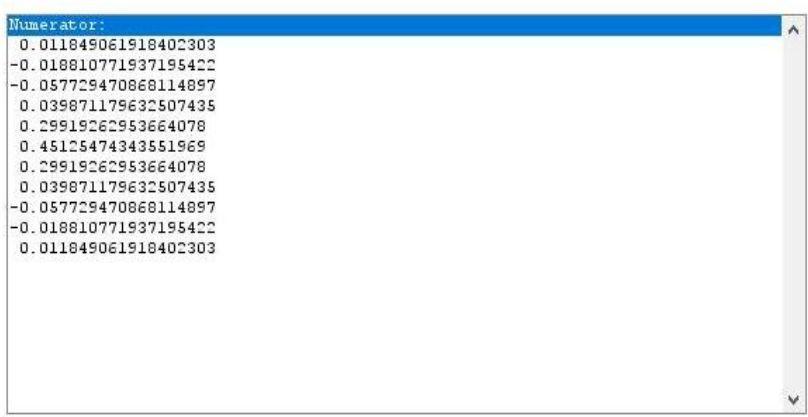

Figure (20): Filter coefficient of Taylor Window

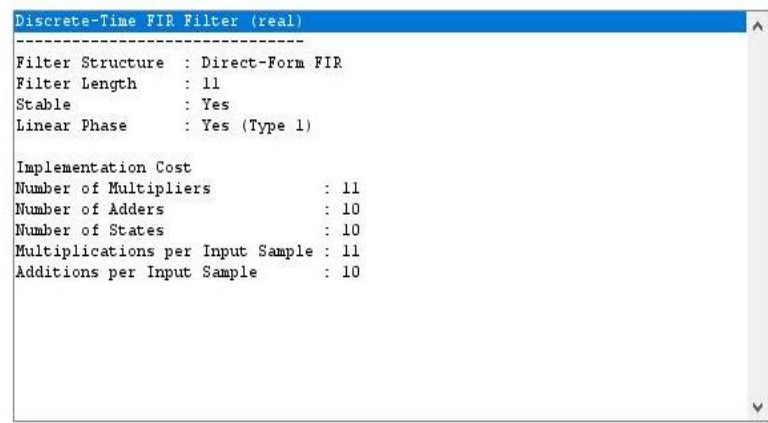

Figure (21): Information of Taylor Window

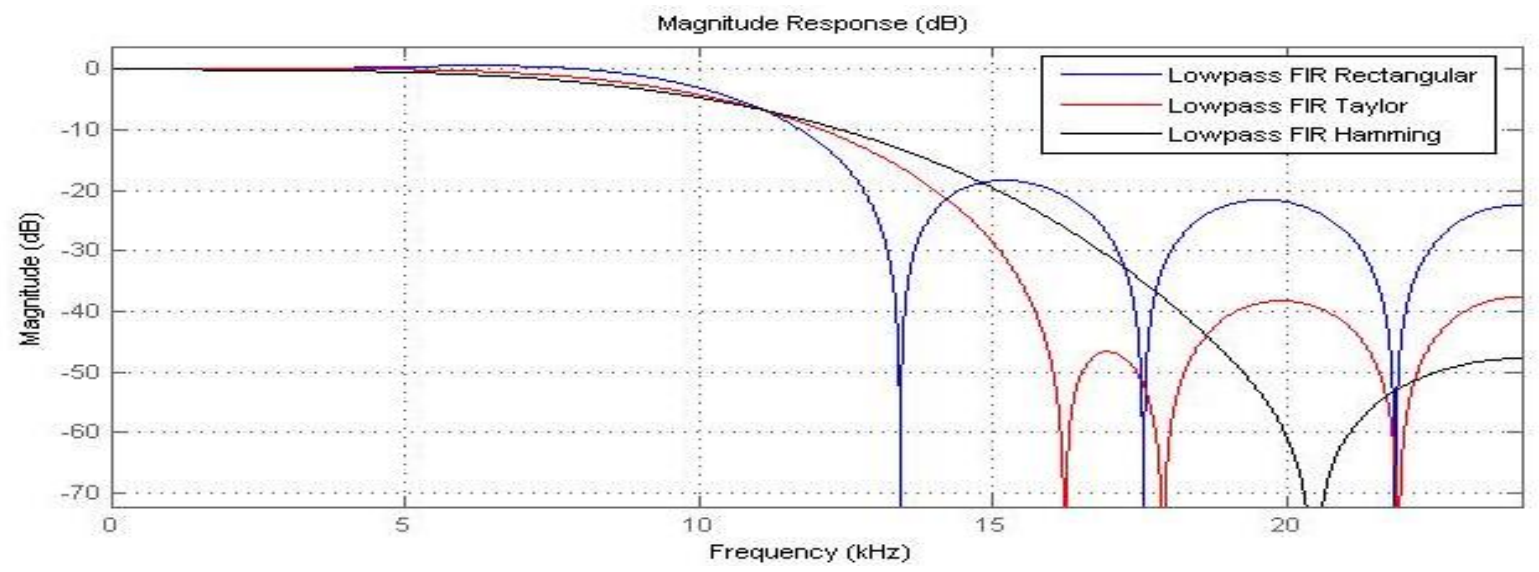

Figure (22): Magnitude respone comparison of Hamming, Rectangular and Taylor window

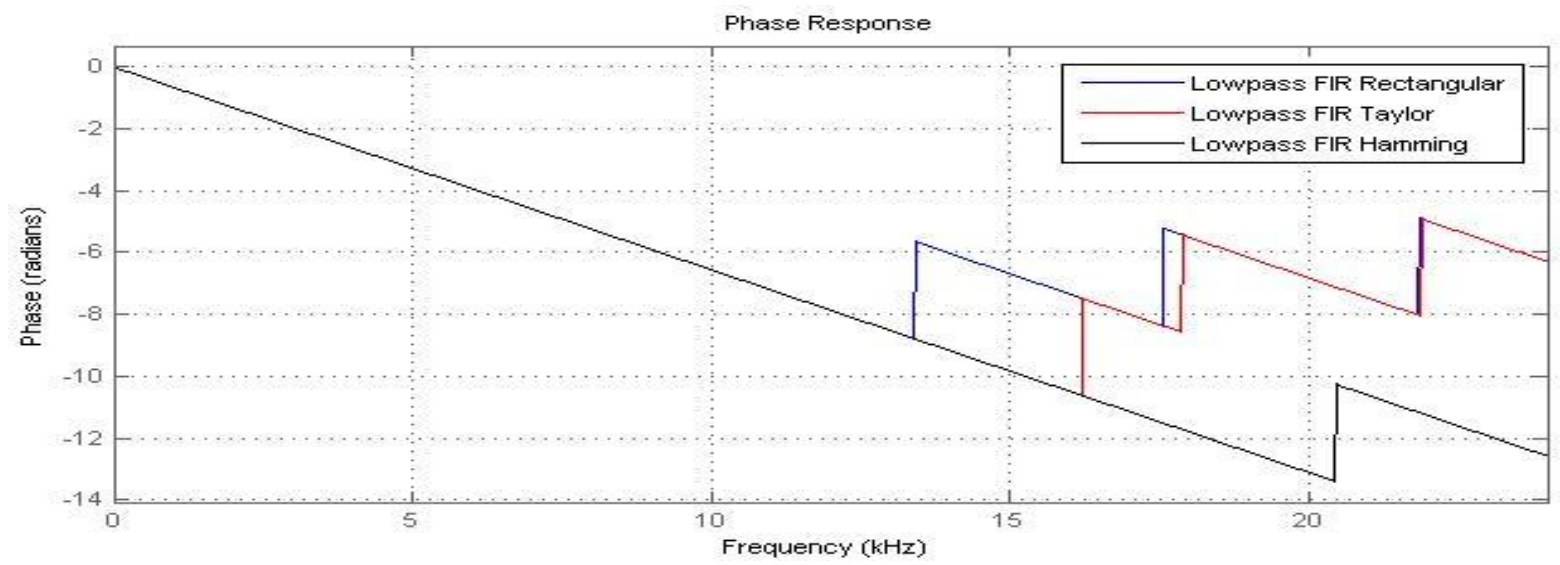

Figure (23): Phase response comparison of Hamming, Rectangular and Taylor window

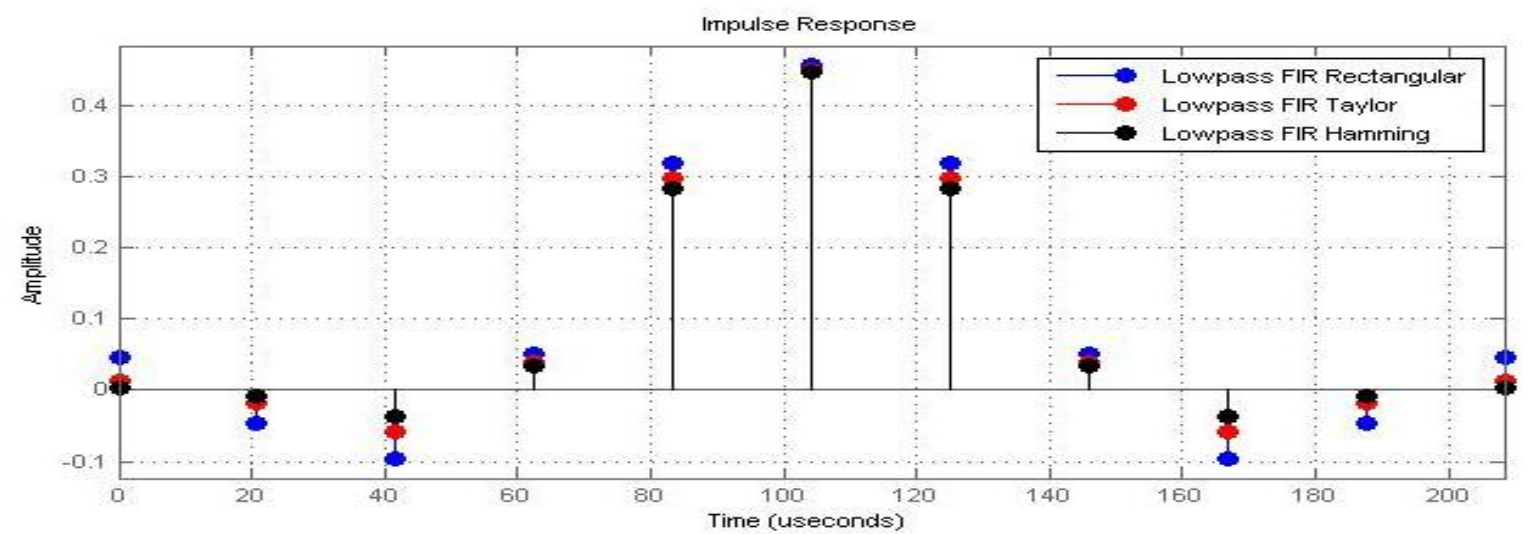

Figure (24): Impulse response comparison of Hamming, Rectangular and Taylor window 


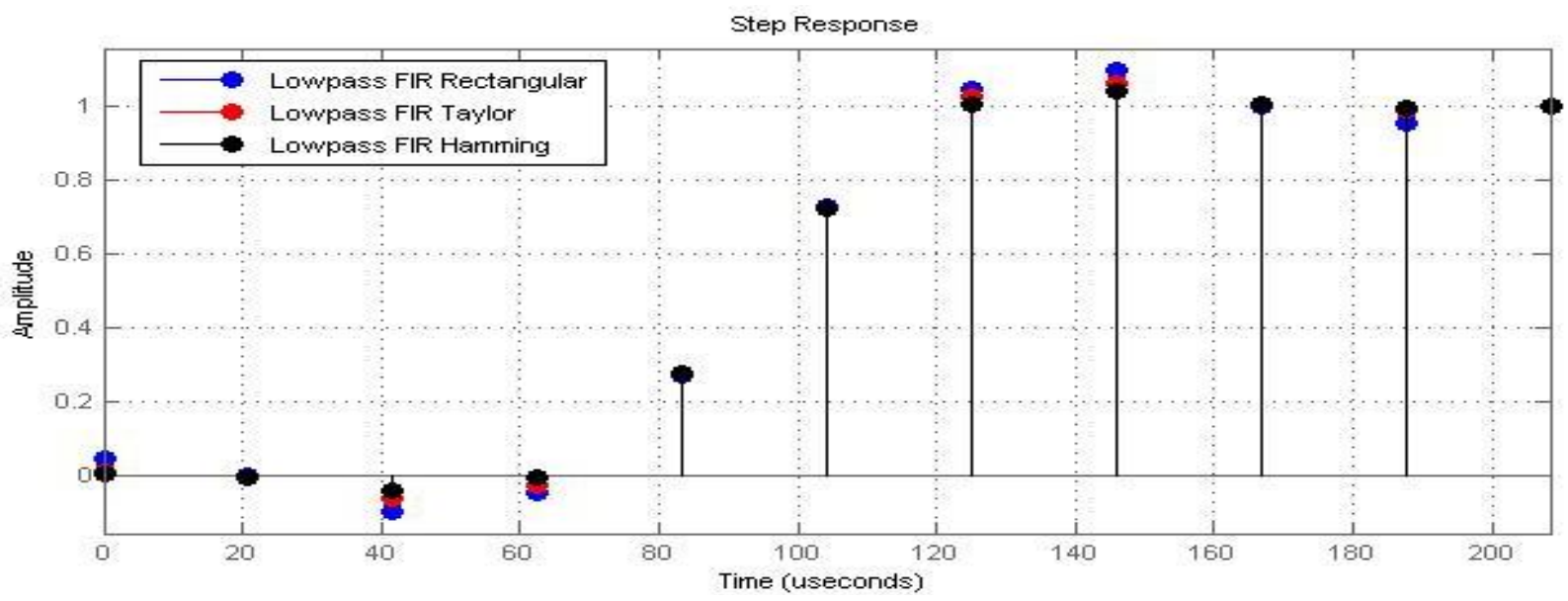

Figure (25): Step response comparison of Hamming, Rectangular and Taylor window

\section{RESULT}

Table (2) Simulation Result in MATLAB

\begin{tabular}{|c|c|c|c|}
\hline Window Technique & Leakage Factor & Relative side lobe attenuation & Mainlobe width (-3dB) \\
\hline Hamming & $0.04 \%$ & $-35.8 \mathrm{~dB}$ & 0.27344 \\
\hline Rectangular & $9.2 \%$ & $-13 \mathrm{~dB}$ & 0.17188 \\
\hline Taylor & $0.34 \%$ & $-29.2 \mathrm{~dB}$ & 0.21875 \\
\hline
\end{tabular}

\section{APPLICATIONS}

From the above simulation result it is found that the Designed FIR filter has lesser main lobe width so it can be used in Speech Processing Applications such as speech filtering, noise reduction, Frequency boosting and Digital Audio Feudalizing etc. In speech filtering; Filter are used to modify the Frequency response of a speech signal according to require applications of speech processing. Designed low-pass FIR filter is used to eliminate the high-frequency spectrum of the speech signal and also as the Designed filter has lesser main lobe width. so it is very useful for spectral analysis of a signal [10]. Signal processing is essential for a wide range of applications from data science to Realtime embedded systems.. MATLAB and Simulink product make it easy to use Signal Processing Techniques to explore and analyze time-series data, and they provide a unified workflow for the development of embedded systems and streaming applications.

\section{CONCLUSION}

In Signal Processing Applications Digital Filters are more preferable than Analog filters. In this report a brief overview of applications of Digital Signal Processing in Radar is presented. Matched filter implementation, echo cancellers and automatic detection and tracking are discussed in separate sections. In most of the modeling, In comparison with real DSP, complex DSP is more abstract and theoretical, but also more powerful and comprehensive. Complex transformation. .and techniques, such as complex modulation, filtering, mixing and adaptive complex etc. are the essence of theoretical DSP. Complex DSP techniques are required for many. wireless high-speed telecommunication standards the performance of each processing were evaluated by comparing many responses such as chirp or step and so on. In this view FIR filter has been designed using Modified coefficient of the Hamming window function and simulated with MATLAB programs. From the simulation results it is observed that the proposed window function produces comparatively much effective type of filter response. In this paper FIR low pass filter has been designed and simulated using Rectangular, Taylor and Hamming Window technique. It has been compared leakage factor, main lobe width and relative side lobe attenuation of the three windows

\section{REFERENCES}

[1] Prof.Gopal S.Gawande, Dr.K.B.Khanchandani, T.P.Marode "performance analysis of fir digital filters design techniques" International Journal of computing \& corporate research volume, 2 issue 1 January 2012.

[2] J.G. Proakis and D. G. Manolakis, Digital Signal Processing Principles, Algorithms and Applications 3rd Edition Prentice- Hall, 2002.

[3] Sanjit K. Mitra, "Digital Signal Processing: A computer-base approach", Tata McGraw-Hill, 2nd Ed, 2001. 
[4] Atul Bhargava, Ravindra Pratap Narwaria;"Design of FIR Digital Filters using Least Square Method Based On Neural Network"; ISSN: 2278 7798; International Journal of Science, Engineering and Technology Research (IJSETR); Volume 1, Issue 5, November 2012.

[5] Sonika Gupta, Aman Panghal; "Performance Analysis of FIR Filter Design by Using Rectangular, Hanning and Bartlett-Hann Windows Methods", Volume 2, Issue 6, June 2012 ISSN: 2277 128X International Journal of Advanced Research in Computer Science and Software Engineering (IJARCSSE). P. Ramesh Babu; "Digital Signal Processing"; Scitech.

[6] Sanjay Sharma; "Digital Signal Processing"; Katson Books.

[7] C. S. Burrus, A. W. Soewito, R. A. Gopinath, "Least squared error FIR filter design with transition bands," IEEE Trans. Signal Process., vol. 40, no. 6, pp. 1327-1340, Jun. 1992.

[8] Wang Yunlong, Wang Shihu, Ji Rendong, “An Extreme Simple Method for Digital FIR Filter Design”, Third International conference on Measuring technology and Mechatronics Automation(ICMTMA),2011,vol 1,pp 410-413.

[9] Fatima, W. "Design and Analysis of Non recursive Digital Filters (FIR)", thesis, University of technology, 1999.

[10] J.F. Kaiser, "Nonrecursive digital filter using the lo-sin h window function," in proc. IEEE int . Symp Circuit Theory, 1974 , pp..20 -23

[11] S. W. A. Bergen and A. Antoniou,'Design of ultraspherical window function with prescribed spectral characteristics," EURASIP Journal on Applied Signal Processing, vol. 13, pp. 2053 - 2065, 2004

[12] Yadwinder Kumar Er. Priyadarshni Dept. of E.C.E. PTU India (Design of Optimum Digital FIR Low Pass Filter Using Hybrid of GA \& PSO Optimization).

\section{BIOGRAPHIES}

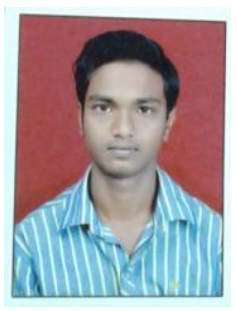

Tarun Kumar Chandra pursuing Bachelor of Engineering in Electrical and Electronics Engineering in 6th semester from Institute of Technology Korba, Chhattisgarh Swami Vivekananda Technical University, Chhattisgarh, India.

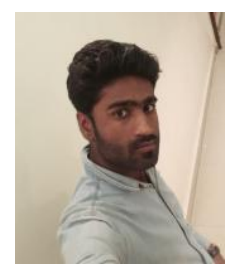

Gautam Sharma pursuing Bachelor of Engineering in Electrical and Electronics Engineering in 6th semester from Institute of Technology Korba, Chhattisgarh Swami Vivekananda Technical University, Chhattisgarh, India.

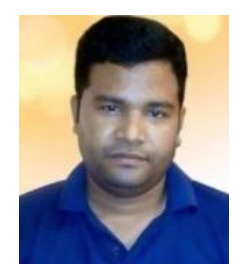

Pranay Kumar Rahi received the Bachelors of Engineering degree in Electronics and Telecommunication Engineering from Government Engineering College, Guru Ghasidas University, Bilaspur, Chhattisgarh, India in 2004,working as a Assistant Professor in Electrical and Electronic Engineering of Institute of Technology Korba since 2008 and pursuing Masters of Engineering in Electronics and Communication Engineering from National Institute of Technical Teacher's Training \& Research, Punjab University, Chandigarh, India. Presently working as assistant professor in Department of Electrical and Electronic Engineering, Institute of Technology Korba, Chhattishgarh since 2008. He has authored More than 40 research publications and published journal papers and research papers in the leading International and National journal. His primary research interest includes Digital Signal Processing, VLSI Design, Control Systems and Digital Electronics and logic design. 